\title{
Enhancing resolution properties of array antennas via field extrapolation: application to MIMO systems
}

\author{
Ruggero Reggiannini
}

\begin{abstract}
This paper is concerned with spatial properties of linear arrays of antennas spaced less than half wavelength. Possible applications are in multiple-input multiple-output (MIMO) wireless links for the purpose of increasing the spatial multiplexing gain in a scattering environment, as well as in other areas such as sonar and radar. With reference to a receiving array, we show that knowledge of the received field can be extrapolated beyond the actual array size by exploiting the finiteness of the interval of real directions from which the field components impinge on the array. This property permits to increase the performance of the array in terms of angular resolution. A simple signal processing technique is proposed allowing formation of a set of beams capable to cover uniformly the entire horizon with an angular resolution better than that achievable by a classical uniform-weighing half-wavelength-spaced linear array. Results are also applicable to active arrays. As the above approach leads to arrays operating in super-directive regime, we discuss all related critical aspects, such as sensitivity to external and internal noises and to array imperfections, and bandwidth, so as to identify the basic design criteria ensuring the array feasibility.
\end{abstract}

Keywords: Uniform linear array; Dense array; Field extrapolation; Angular super-resolution; Super-directive array; Multiple beamforming; Multipath channel; MIMO systems

\section{Introduction}

One of the most appealing features of multiple-input multiple-output (MIMO) systems stands in their capability to exploit the structure of a multipath propagation channel to multiply the transmission rate between two multiantenna terminals $[1,2]$. Actually, when the propagation environment is rich of backscattering obstacles, it is likely that there exist several independently fading paths linking pairs of antenna elements between the transmitter and the receiver arrays, thus potentially creating margins for spatial multiplexing gain.

It is known that this gain cannot exceed the minimum between the numbers of spatially orthogonal ${ }^{1}$ beams that can be implemented by means of the transmitting and receiving arrays. Specifically, for a uniform linear array ${ }^{2}$ (ULA) with isotropic elements, the maximum achievable number of approximately orthogonal beams is equal to twice the array length normalized to the wavelength,

Correspondence: ruggero.reggiannini@iet.unipi.it

Department of Information Engineering, University of Pisa, Via G. Caruso 16, 56122 Pisa, Italy since the angular resolution allowed by the array, in the directional cosine domain $(-1,1)$ is roughly equal to the inverse of the normalized array length [3, Chap. 7].

This bound to the number of beams can actually be attained provided that the element spacing does not exceed half wavelength (critical spacing). For larger-than-critical spacing, the number of available orthogonal beams is smaller than the above limit, while, on the other hand, for a ULA of fixed length, the angular resolution cannot be improved by arranging more elements at less than the critical distance. These observations seem to suggest that the most convenient spacing to adopt in a linear array is the critical one.

One of the purposes of this paper is to revamp the above issues and re-discuss the above conclusion, showing that, when tighter-than-critical spacing is used along with unconventional non-uniform element weighing, there exist further design margins allowing the formation of a larger number of more directive beams with respect to the conventional uniform-weighing approach. These over-performing beams can be designed so as to

\section{Springer}


be approximately orthogonal to one another and to uniformly cover the entire horizon. The prospective advantages offered by such "dense" arrays are evident in those scenarios where the angular resolution of a conventional fixed-length ULA is coarser than the angular extension of scatterers surrounding the terminals, i.e., where potential margins exist for a capacity enhancement, that could be achieved if only more directive arrays were available.

With reference to a receiving array, the approach followed here stems from the basic observation that the field received at all points of a straight line in threedimensional space as a result of remote sources, viewed as a function of a single spatial coordinate, is strictly band-limited, being limited the horizon of possible directions of arrival of the field components. Accordingly, we can apply to it all results known for band-limited functions, in particular, the noteworthy property that these functions are analytical, i.e., such that their exact knowledge in a finitelength interval allows, in principle, their extrapolation over the entire domain of definition, having infinite extension [4-9], thus potentially prospecting the achievement of unlimited angular resolution. As extensively discussed in these references, however, the implementation aspects, such as the need to sample the field by means of a sensor array, with consequent loss of information due to the finiteness of the spatial sampling frequency, and the errors inherent in the analog-to-digital conversion and in the array implementation, along with other limiting factors, make the above extrapolation process reliable only within a limited range in the vicinity of the array.

In the following, we investigate the above issues and show that under certain conditions it is possible to extrapolate the received field so as to obtain a virtual (actual plus extrapolated) array significantly longer than the real one and making it possible to improve on its original angular discrimination capability. Resorting to conventional Fourier transformation techniques, we show that the virtual array thus obtained can be associated with an augmented set of narrower beam patterns able to cover the entire horizon. Since the transformation linking the actual field samples produced by the array elements to the extrapolated data is linear, the above narrower beam patterns can be implemented by applying to the actual sensor array a set of weighing windows represented by the transformation coefficients. The results are also applicable to a transmit array using an inverse beamforming scheme with the same weighing windows.

We note that many authors have proposed algorithms for extrapolating electromagnetic or acoustic fields, but these schemes are commonly based on the spatial sampling of a wavefront generated by a specific source and on the application of Huygens' principle for estimation of the field over previous or successive wavefronts (see, e.g., $[10,11])$. Conversely, with regard to the approach pursued here, there seem to be no examples of extrapolation of the field received at the points of a straight segment (and due to remotely distributed sources) beyond the limits of the segment itself.

Our approach is reminiscent of techniques already known in the literature as super-resolution or superdirectivity, even though the application framework, the constraints, and the objectives pursued here are definitely different. In the literature, the mentioned techniques have been addressed with reference to different categories of problems. On the one hand, super-resolution algorithms have been studied extensively in the past in conjunction with ingenious extrapolation schemes to improve the discrimination capability of specific instrumentation, such as optical sensors or spectrum analyzers, in response to one- or two-dimensional band-limited signals observed in truncated intervals [4-9]. In these references the interest of researchers is mainly focused on the extrapolation schemes and the relevant performance limits, with no reference to their possible applications to beamforming issues. On the other hand, the potential super-directive properties of dense arrays are long since known, and super-directivity is defined mathematically as the solution to an optimization problem, namely, the search of the weighing window maximizing the array directivity for a fixed array response in the steering direction [12, Sect. 9.2.1 and overview notes in Sect. 9.1]. Similar concepts are also found in the theory underlying the Capon beamformer and variants thereof [13, Chap. 6] and in the immense literature dealing with super-directivity issues (examples of recent papers are in [14], e.g., [15] and [16], or [17, 18], just to cite different contexts).

Furthermore, although it is well known that superdirectivity is only viable with less-than-critical element spacing, no specific mention was found in the literature as to the possible relationship between the superdirective behavior of the array and its ability to extrapolate the received field. In particular, up to the author's knowledge, no approach based on field extrapolation has been proposed thus far as an aid for the design of multibeam systems with a controlled degree of superdirectivity.

In any case, as mentioned earlier, when dealing with super-directive beams, it is necessary to carefully evaluate the impact of super-directivity on the output noise level and on the beam bandwidth, as well as the sensitivity of the beam response to perturbations of the weighing coefficients, since the above factors rapidly tend to deteriorate the beam performance [12, Sect. 9]. This paper takes into account all these aspects and formulates simple design criteria to identify technologically feasible trade-off solutions, capable to provide significant performance gains 
compared to a conventional uniformly weighted scheme. The main conclusion is that it is possible to increase the number of nearly orthogonal beams with which to cover the horizon, and then ultimately boost the capacity achievable by a hypothetical MIMO system incorporating the above technique, in those cases where the environment cooperates. We note that, although this paper has been written having in mind wireless MIMO applications, the proposed approach can also prove useful in different areas, such as radar, sonar, seismic, space exploration, and the like.

The paper is organized as follows: Section 2 provides the system overview and illustrates the proposed novel approach to extrapolate a receiving array, pointing out conditions allowing extrapolation. Section 3 discusses the main factors affecting feasibility and performance of a super-directive beamformer based on field extrapolation, while Section 4 presents the criteria to be used for the design of a multibeam system suitable to cover the horizon with improved angular resolution. Conclusions are in Section 5.

\section{System overview and extrapolation technique}

Throughout the paper, we refer to a narrow-band ULA made up of $M$ identical isotropic point elements operating at the frequency $f_{0}$ and arranged along the $x$-axis, whose scale is normalized with respect to the wavelength $\lambda$ (Fig. 1). In this normalized scale, the distance between the elements is denoted as $\Delta$ and the array effective length (accounting for its electromagnetic properties) is $L=M \Delta$ while its physical length is $L_{\mathrm{a}}=(M-1) \Delta$. Without loss of generality, in the following, we consider a receiving array immersed in the field generated by a plurality of remote sources (ideally, located at infinite distance from the array) distributed over the horizon.

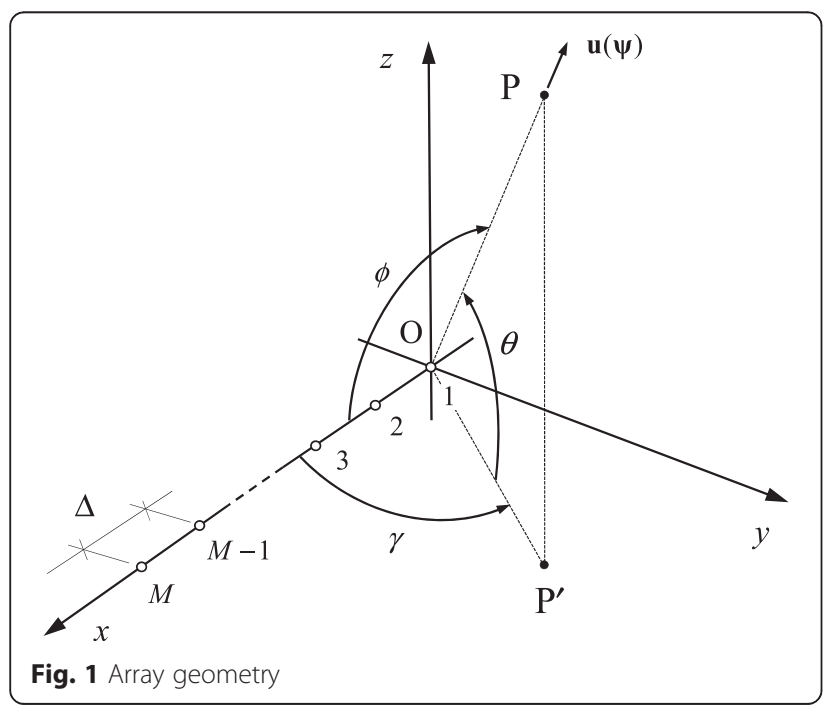

The above sources can be either potentially beneficial as, for example, when they are due to scatterers distributed in the environment that reflect or diffuse useful signal components, or they may represent sources of noise or interference, whose effect is to be considered deleterious for the communication system. In any case, let $\vec{e}(x)$ denote the electrical field on the points of the $x$-axis (to which we restrict our attention), resulting from the combination of the cited components.

\subsection{Spectrum of $\vec{e}(x)$}

We start from the observation that $\vec{e}(x)$ is a bandlimited function of $x$, since its Fourier transform (FT) can be nonzero only within the interval $(-1,1)$ of the cosines of real directions. If $d v_{0}(\boldsymbol{\psi})$ indicates the output, in phasor notation, of a hypothetical array element located at $x=0$ in response to the electrical field $d \vec{e}_{0}(\boldsymbol{\psi})$ arriving from the solid-angle direction $\boldsymbol{\psi} \equiv(\gamma, \theta),{ }^{3}$ the output resulting from all field components in $x=0$ is given by

$$
v_{0} \triangleq v(x=0)=\oint d v_{0}(\boldsymbol{\Psi})
$$

where the integral is taken on the overall threedimensional (3D) horizon. In consequence, the phasor field received at a generic point of abscissa $x$ reads as

$$
v(x)=\oint \exp [-j 2 \pi x \mathbf{i} \cdot \mathbf{u}(\boldsymbol{\Psi})] d v_{0}(\boldsymbol{\Psi})
$$

where $\mathbf{i}$ is the $x$-axis versor and $\mathbf{u}(\psi)$ is the versor associated to the direction $\psi$. To be specific, we consider the function $v(x)$ for the following two noteworthy cases, for which (2) can be easily calculated:

1. The remote sources can be modeled as a uniform uncorrelated continuum for all directions of the $3 D^{4}$ horizon. In such a case, it is known [13, Chap. 5] that $v(x)$ is a wide-sense stationary process and its autocorrelation $R_{v}(\delta x)$ depends on the difference $\delta x \triangleq x_{2}-x_{1}$ between the abscissas of the points considered, as follows

$R_{\nu}(\delta x)=\sigma_{v}^{2} \operatorname{sinc}(2 \delta x)$

where $\operatorname{sinc}(x) \triangleq \sin (\pi x) /(\pi x)$ and $\sigma_{v}^{2}$ is the mean square value of $v(x)$. The FT of (3) is

$R_{v}(\delta x) \stackrel{F}{\rightleftarrows} \frac{\sigma_{v}^{2}}{2} \operatorname{rect}\left(\frac{\Omega}{2}\right)$ 
where $\operatorname{rect}(x)$ equals unity in $(-0.5,0.5)$ and is zero elsewhere, and $\Omega=\cos \phi$ is the variable representing the (transformed) domain of directional cosines.

2. The remote sources can be modeled as a uniform uncorrelated continuum for all directions of the horizontal plane $e^{5} \mathrm{Oxy}$, and there are no sources in the other directions. This model could be considered realistic when the predominant external disturbance is interference generated by man-made devices, as might occur for wireless telecommunication networks distributed over the territory [19]. In such a case, the direction of arrival (DOA) of the field components is identified by a scalar $\gamma \equiv \phi$ (since $\psi=(\gamma, 0)$ ) (Fig. 1); and hence from (2), it is found [13, Chap.0 5]):

$$
R_{v}(\delta x)=\sigma_{v}^{2} J_{0}(2 \pi \delta x)
$$

where $J_{0}(\cdot)$ is the first-kind Bessel function of zero order and the other terms retain the same meaning already specified for (3). The FT of (5) is now

$$
R_{v}(\delta x) \stackrel{F}{\rightleftarrows} \frac{\sigma_{v}^{2}}{\pi} \frac{\operatorname{rect}(\Omega / 2)}{\sqrt{1-\Omega^{2}}}
$$

\subsection{Extrapolation of $v(x)$}

As pointed out in [4-9], a low-pass band-limited function such as $v(x)$ is analytic and therefore, in principle, it can be extrapolated over the entire $x$-axis provided that it is exactly known at all points of a finite-length interval. In practice, this possibility is impaired by the presence of inaccuracies (due to internal receiver noise, quantization noise, etc.) in the knowledge of the above function and also by the need to adopt a discrete representation of lengths and angles suitable for application of digital signal processing techniques.

Under certain circumstances, however, it is possible to take advantage of the above property, as will be shown shortly. Turning to a space-discrete representation of $v(x)$, we assume this function is sampled at $M$ evenly spaced points with normalized spacing $\Delta$, just as it would be done by the elements of a ULA, that are capable to take snapshots of the field present at the points where they are positioned. Our aim here is to get knowledge of $v(x)$ over an extended set of $N \Delta$-spaced points, with $N>M$, through extrapolation of the missing $N-N$ values from the known $M$ samples. To proceed, we denote $\mathbf{x}=\left(\begin{array}{llll}x_{0} & x_{1} & \cdots & x_{N-1}\end{array}\right)^{t}$, where the superscript ${ }^{t}$ indicates transpose, the vector of abscissas $x_{n}=n \Delta, n=0,1, \cdots, N-1$, to which the samples generated by the extrapolation algorithm are to be orderly associated, as indicated in Fig. 2. The vector resulting from the extrapolation process is denoted as $\tilde{\mathbf{v}}=\left(\begin{array}{lllll}\tilde{v}_{0} & \tilde{v}_{1} & \cdots & \tilde{v}_{N-1}\end{array}\right)^{t}$, and it incorporates as sub-vector the set of the original $M$ samples, such that $\tilde{v}_{n}=v\left(x_{n}\right)$, arranged midway in $\tilde{\mathbf{v}}$ at the positions with indices comprised between $\lfloor(N-M+1) / 2\rfloor$ and $\lfloor(N+M-1) / 2\rfloor,\lfloor z\rfloor$ denoting the greatest integer not exceeding $z$.

It is worth observing that the finiteness that must be imposed on the length of any actual extrapolation window, together with the discretization of the $x$ coordinates, prevents the FT of the extrapolated sequence from being exactly zero outside the range of the real directions, due to the spectral ripple introduced by the cited truncation. This type of errors concurs to limit the accuracy and range of the extrapolation algorithms to be shortly derived.

The approach we follow starts from the observation that a ULA of $N$ elements can be associated to $N$ basis directions equally spaced in the cosine domain, on any plane containing the array (e.g., [3, Chap. 7])

$$
\Omega_{k} \triangleq \cos \phi_{k}=\frac{1}{\Delta} \frac{k}{N}, \quad k=-\left\lceil\frac{N}{2}-1\right\rceil, \cdots-1,0,1, \cdots,\left\lfloor\frac{N}{2}\right\rfloor
$$

where $\phi_{k}$ is the angle between the $x$-axis and the $k$ th direction, measured with respect to the positive direction of the $x$-axis (see Fig. 2) and $\lceil z\rceil$ denotes the smallest integer not smaller than $z$. The basis directions (7) are used to build the Fourier orthonormal basis for the space of $\mathrm{N}$-dimensional complex vectors, as follows

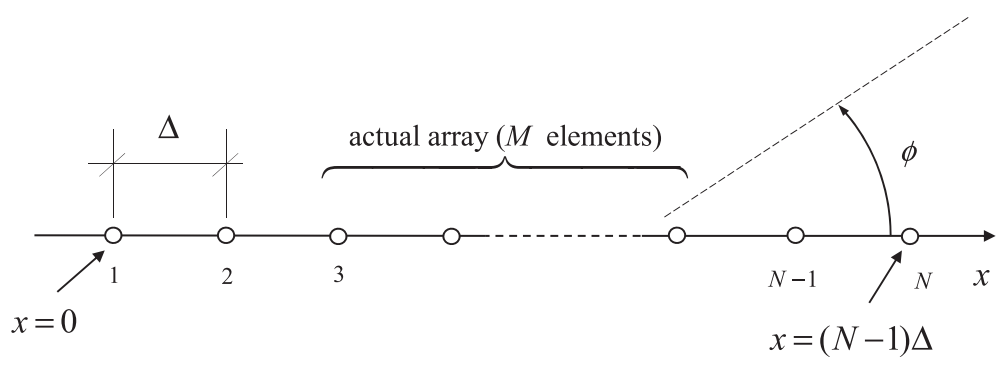

Fig. 2 Arrangement of elements of true and virtual arrays 


$$
\begin{aligned}
& \mathbf{u}_{k}=\frac{1}{\sqrt{N}}\left(\begin{array}{lllll}
1 & e^{-j 2 \pi \Delta \Omega_{k}} & e^{-j 2 \pi 2 \Delta \Omega_{k}} & \cdots & \left.e^{-j 2 \pi(N-1) \Delta \Omega_{k}}\right)^{t},
\end{array}\right. \\
& k=0,1, \cdots, N-1
\end{aligned}
$$

where the range of variation of the index $k$ has been rearranged keeping into account the periodic properties of the exponentials in (8). ${ }^{6}$ We recall that the unit vectors (8) can be stacked so as to form the unitary matrix

$$
\mathbf{F} \triangleq\left(\begin{array}{llll}
\mathbf{u}_{0} & \mathbf{u}_{1} & \cdots & \mathbf{u}_{N-1}
\end{array}\right)^{t}
$$

which is recognized to be the FT matrix, mapping the spatial domain of the discrete $x$ coordinates onto the domain of the discrete $\Omega$ directional cosines.

To create a useful edge for extrapolation, it is necessary that the spatial sampling rate exceeds the Nyquist critical rate of two samples per wavelength, i.e.,

$$
\frac{1}{\Delta}>2
$$

since only the fulfillment of this condition may give rise to a dependency between the field samples that can be exploited to achieve our purpose. Assuming (10) holds true, it is seen that part of the cosines in (7), namely those associated to values of $k$ close to the ends of the interval $(-\lceil N / 2-1\rceil,\lfloor N / 2\rfloor)$ become greater than one in absolute value, i.e., the corresponding directions $\phi_{k}$ are not real. This permits to argue that, having a snapshot from a $N$-element array available, its projections on these directions must be close to zero, even though not exactly zero in view of the spectral leakage induced by the finite sampling rate and array length. In other terms, denoting as $\mathbf{v}=\left(\begin{array}{llll}v_{0} & v_{1} & \cdots & v_{N-1}\end{array}\right)^{t}$, with $v_{n}=v\left(x_{n}\right), n=$ $0,1, \cdots, N-1$, the vector of the actual field values, that would be provided by a hypothetical $N$-element array, ${ }^{7}$ and as $\mathbf{V}=\mathbf{F v}$ its FT, the components of $\mathbf{V}$ are approximately zero at those entries corresponding to non-real directions, i.e., the following must hold true

$$
V_{k} \approx 0, \quad\lfloor\Delta N\rfloor<k<N-\lfloor\Delta N\rfloor
$$

while the components of $\mathbf{V}$ are generally non-zero on the remaining directions, identified in (8) by the values of $k$ such that: $0 \leq k \leq\lfloor\Delta N\rfloor, N-\lfloor\Delta N\rfloor \leq k \leq N-1$. It is worth pointing out that conditions (11) do not depend on the values of the received field but descend solely from considerations based on the physical properties of the scenario.
Defining $\beta \triangleq\lfloor\Delta N\rfloor$ and assuming that the components $V_{k}$ in (11) are exactly zero, it is seen that in the vector $\mathbf{V}$, there only remain $Q \triangleq 2 \beta+1$ degrees of freedom, equal to the number of its left (possibly) nonzero components. In consequence, knowledge of the field samples $v\left(x_{n}\right)$ on the $M$ central positions indicated in Fig. 2 is sufficient to know the entire vector $\mathbf{V}$, provided that

$$
M \geq Q
$$

Indeed, assuming (12) holds true and the components $V_{k}$ in (11) are zero, it is possible to formulate the non-homogeneous linear system of $M$ equations in $Q$ unknowns

$$
\overline{\mathbf{F}}_{i} \overline{\mathbf{V}}=\overline{\mathbf{v}}
$$

where $\overline{\mathbf{v}}$ and $\overline{\mathbf{V}}$ are shortened versions of vectors $\mathbf{v}$ and $\mathrm{V}$, respectively, as follows

$$
\begin{aligned}
& \overline{\mathbf{v}}=\left(\begin{array}{llllll}
v_{\lfloor(N-M+1) / 2\rfloor} & \cdots & v_{\lfloor(N+M-1) / 2\rfloor}
\end{array}\right)^{t} \\
& \overline{\mathbf{V}}=\left(\begin{array}{llllll}
V_{0} & \cdots & V_{\beta} & V_{N-\beta} & \cdots & V_{N-1}
\end{array}\right)^{t}
\end{aligned}
$$

and $\overline{\mathbf{F}}_{i}$ is a $M \times Q$ matrix obtained from the inverse Fourier transform (IFT) matrix $\mathbf{F}^{-1}$ by deleting the columns of indices ranging from $\beta+1$ to $N-\beta-1$ and retaining only the rows of indices between $\lfloor(N-M+1) / 2\rfloor$ and $\lfloor(N+M-1) / 2\rfloor$. Actually, conditions (11) only hold approximately, so that in (13) we should replace the vector $\overline{\mathbf{V}}$ containing true samples of the FT of $\mathbf{v}$ with the vector $\tilde{\overline{\mathbf{V}}}$, denoting an approximation of $\overline{\mathbf{V}}$. For the specific case $M=Q$, the system (14) has a unique solution, i.e.,

$$
\tilde{\overline{\mathbf{V}}}=\overline{\mathbf{F}}_{i}^{-1} \overline{\mathbf{v}} \text {. }
$$

We observe that the inverse matrix in (16) exists being non-zero all minors of a FT matrix deriving from circularly adjacent rows or columns [20, p. 8], but it tends to become ill-conditioned as $\Delta$ or the ratio $M / N$ decreases. This aspect must be carefully considered when designing the system (Sections 3 and 4). It is also noted that (16) actually defines a multiple beamformer, since the vector of observations $\overline{\mathbf{v}}$ projected onto the rows of the matrix at the right-hand side yields the array response to the field components coming from the directions associated with the entries of $\tilde{\overline{\mathbf{V}}}$. This point will be pursued further in Section 4.

If $M>Q$, the number of constraints in the system (13) exceeds the number of unknowns (overdetermined problem), and in this case, a reasonable approach is to replace $\overline{\mathbf{F}}_{i}^{-1}$ in (16) with the pseudo-inverse of $\overline{\mathbf{F}}_{i}$, defined as [13, p. 1371] 


$$
\overline{\mathbf{F}}_{i}^{\dagger} \triangleq\left(\overline{\mathbf{F}}_{i}^{H} \overline{\mathbf{F}}_{i}\right)^{-1} \overline{\mathbf{F}}_{i}^{H}
$$

where the superscript $H$ denotes conjugate transpose. The same considerations already made for $\overline{\mathbf{F}}_{i}^{-1}$ also hold for $\overline{\mathbf{F}}_{i}^{\dagger}$.

From the above discussion, it is possible to outline the extrapolation procedure, envisaging the following steps:

1. We start from a snapshot $\overline{\mathrm{v}}$ at the output of the $M$-element ULA with normalized element spacing $\Delta$ and effective length $L=M \Delta$;

2. Leaving $\Delta$ unchanged, we fix the number of positions $N>M$ on which to obtain the vector resulting from extrapolation, i.e., we fix the length $N \Delta$ of a hypothetical ULA incorporating the actual ULA (criteria for choosing $N$ are given in Section 4);

3. From (16) to (17), we compute the $Q$ - element vector $\tilde{\overline{\mathrm{V}}}$ after pre-calculating the matrix of coefficients $\overline{\mathrm{F}}_{i}^{-1}$ or $\overline{\mathrm{F}}_{i}^{+}$;

4. We construct the $N$ - element vector $\tilde{\mathbf{V}}$ by inserting $N-Q$ zero entries after the first $\beta+1$ elements of $\tilde{\overline{\mathrm{V}}}$;

5. We take the IFT so as to obtain the vector $\tilde{\mathbf{v}}=\mathrm{F}^{-1} \tilde{\mathbf{V}}$ representing the result of the extrapolation procedure whose $M$ central entries coincide with $\overline{\mathrm{v}}$.

\subsection{Example}

We refer to the 2D field model introduced at point 2 of Section 2.1. Random realizations of the field are generated by combining the effects of numerous remote point sources, whose contributions are assumed independent identically distributed (IID) circular Gaussian RVs. We let $\Delta=0.1, M=9$, and $N=21$. From the foregoing discussion, we find $\beta=2, Q=5$, and hence in the FT of the received field, we have to insert $N-Q=16$ central zeros. Figure 3 shows a typical noiseless realization of the real part of the received field $\mathbf{v}$ along with the FT of $\mathbf{v}$ taken on 21 samples (solid lines; the vertical scales are arbitrary), while the diamonds represent the real part of $\overline{\mathbf{v}}$ at the output of a nine-element array, i.e., a truncated version of $\mathbf{v}$, together with its 21-point FT, and finally the dashed lines with triangles are the result of the extrapolation procedure. The imaginary part of the field has the same statistical behavior as the real part in view of the above assumptions. As is seen from the upper diagram, the extrapolated field closely follows the actual field up to a certain distance from the array ends, then it begins to exhibit some deviations, mainly to be ascribed to the approximations in (11). Such deviations tend to worsen as $N / M$ and/or $\Delta$ grows, and also when the observed field samples, assumed noiseless thus far, are affected by internally generated additive noise.
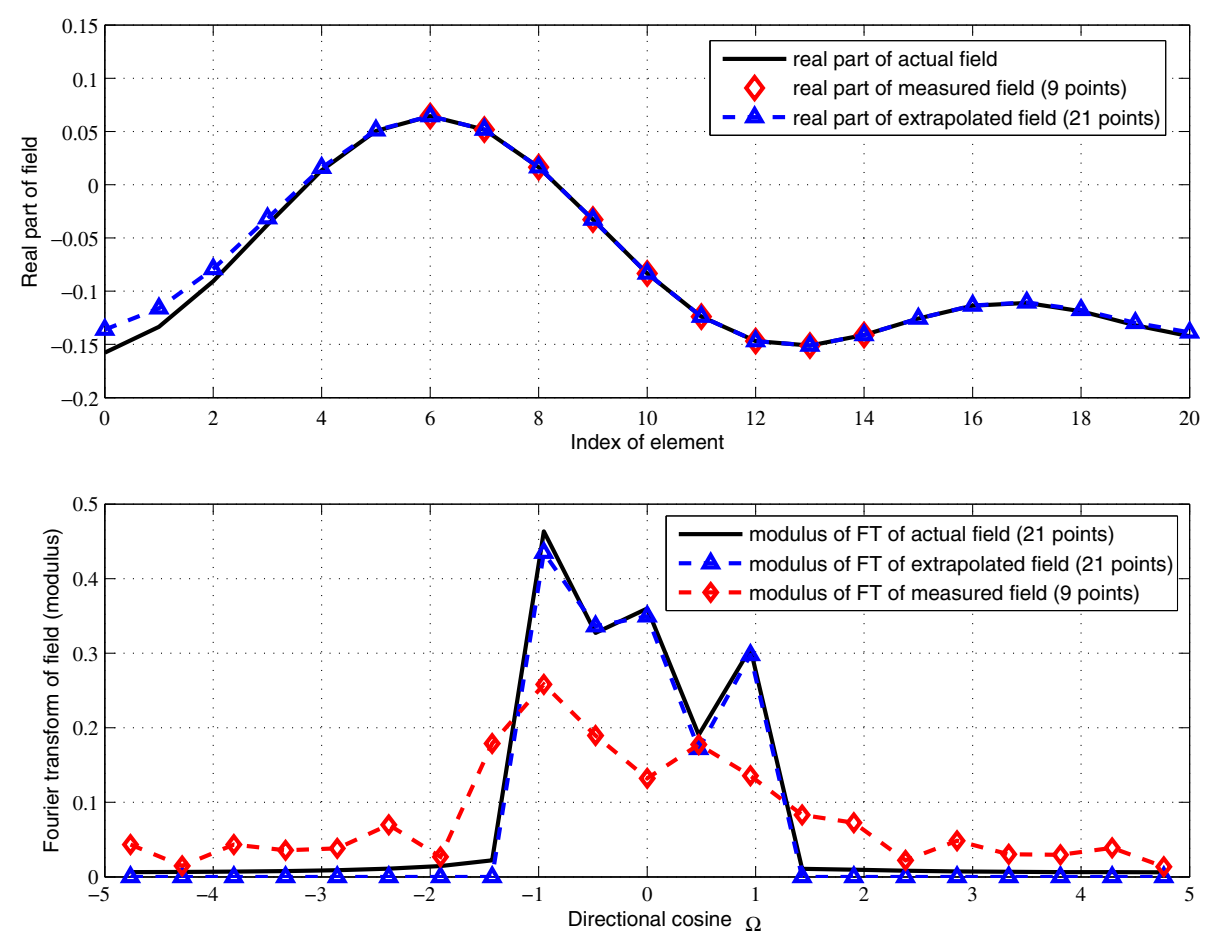

Fig. 3 Example of field extrapolation. Arbitrary vertical scales. Upper diagram: field; lower diagram: spectrum. $\Delta=0.1, M=9, N=21$ 


\subsection{Discussion}

In the foregoing example, the transformation matrix (17)

takes on the following values-

\begin{tabular}{|c|c|c|c|c|c|c|c|c|c|}
\hline \multirow{5}{*}{$\overline{\mathbf{F}}_{i}^{\dagger}=$} & 14.13 & -13.95 & -9.58 & 5.354 & 12.67 & 5.354 & -9.58 & -13.95 & 14.13 \\
\hline & $10.27-\mathrm{j} 0.6065$ & $-10.78-\mathrm{j} 1.618$ & $-7.598-j 0.1122$ & $3.563+j 1.421$ & $9.14+j 1.378$ & $3.824-\mathrm{j} 0.3073$ & $-7.293-j 2.132$ & $-10.77-\mathrm{j} 1.631$ & $9.64+j 3.61$ \\
\hline & $3.374-\mathrm{j} 0.4366$ & $-4.162-j 0.828$ & $-2.862+j 0.4055$ & $1.428+j 1.435$ & $3.734+\mathrm{j} 1.152$ & $1.988-\mathrm{j} 0.3812$ & $-2.136-j 1.947$ & $-3.905-j 1.66$ & $-j 2.26$ \\
\hline & $3.374+j 0.4366$ & $-4.162+j 0.828$ & $-2.862-j 0.4055$ & $1.428-j 1.435$ & $3.734-\mathrm{j} 1.152$ & $1.988+\mathrm{j} 0.3812$ & $-2.136+\mathrm{j} 1.947$ & $-3.905+\mathrm{j} 1.66$ & $2.54-j 2.26$ \\
\hline & $10.27+j 0.6065$ & $-10.78+\mathrm{j} 1.618$ & $-7.5976+\mathrm{j} 0.1122$ & $3.563-j 1.421$ & $9.14-j 1.378$ & $3.824+j 0.3073$ & $-7.293+\mathrm{j} 2.132$ & $-10.77+\mathrm{j} 1.631$ & $9.64-j 3.61$ \\
\hline
\end{tabular}

whose rows can be regarded as beamforming weighing windows with nominal looking directions $\pi / 2, \pm$ arc $\cos (1 / 2.1)$, and $\pm \arccos (2 / 2.1)$, respectively. Inspection of the values of the entries in (18), in particular of those with alternating signs in the first row (associated to the broadside direction), indicates that the array is being operated in super-directive conditions, with a degree of super-directivity depending on the choice of parameters involved in the extrapolation procedure. Actually, it can be verified (see Tables 1 and 2 in Section 4) that the array gain associated to the rows of (18) exceeds that achievable by uniform weighing though it does not reach the maximum possible value envisaged in [12, Sect. 9.2.1]. This behavior is not surprising as many of the band-limited extrapolation algorithms proposed in the literature (e.g., $[4,6])$ are actually aimed at achieving super-resolution in the spectral domain. In our context, this is equivalent to achieving super-directivity in the angular domain by extrapolating the values taken by the field beyond the physical extension of the array.

An interesting aspect that is worth pursuing relates to the properties of the beam patterns that are implicitly generated by the rows of the transformation matrix in (16) or (17). Actually, it can be shown that through a proper choice of the parameters $M, \Delta$, and $N$, the number $Q$ of these beams and their directivity can easily exceed those achievable through uniform-weighing beamforming applied to critically spaced arrays of equal length. It seems therefore appropriate to investigate the conditions under which these beams retain the properties of being approximately orthogonal and capable to cover the entire horizon. Prospectively such an augmented set of beams can boost the spatial multiplexing properties of the MIMO communication system, provided that the environment surrounding the array antennas is sufficiently rich of obstacles with angular size comparable to or smaller than the enhanced angular discrimination capability of the array. This approach to beamforming will be referred to in the following as field extrapolation (FE)based beamforming.

As mentioned earlier, an array operating in superdirective mode has to face peculiar issues, such as a pronounced sensitivity to electro-mechanical imperfections and errors in the implementation of the beamforming weights, as well as increased vulnerability to internal noise, in comparison with a conventional uniformly weighted beamformer. In addition, when a superdirective array is operated in the active mode, the

Table 1 Performance indices for the FE beam patterns of the examples

\begin{tabular}{|c|c|c|c|c|c|c|c|c|c|}
\hline Parameters $(M, \Delta, N)$ & Number of beam patterns & $G_{E}(3 D)(d B)$ & $G_{E}(2 \mathrm{D})(\mathrm{dB})$ & $G_{n}(\mathrm{~dB})$ & $\gamma_{E}(3 D)$ & $\gamma_{E}(2 \mathrm{D})$ & $\gamma_{n}$ & $Q_{b w}(3 D)$ & $Q_{b w}(2 D)$ \\
\hline $9,0.1,21$ & 5 & 5.4 & 6.8 & -17.5 & 0.13 & 0.20 & 0.45 & 200 & 270 \\
\hline $8,0.25,12$ & 7 & 6.8 & 7.9 & -11.7 & 0.07 & 0.13 & 0.22 & 71 & 92 \\
\hline $3,0.25,4$ & 3 & 3.0 & 4.6 & 3.0 & 0.0039 & 0.0050 & 0.014 & 1 & 1.4 \\
\hline $5,0.125,8$ & 3 & 3.0 & 4.6 & 3.2 & 0.0054 & 0.0071 & 0.024 & 0.96 & 1.4 \\
\hline $5,0.125,16$ & 5 & 5.0 & 6.2 & -37.5 & 1.4 & 2.4 & 3.3 & 18,000 & 24,000 \\
\hline $6,0.125,16$ & 5 & 5.1 & 6.4 & -27.0 & 0.39 & 0.68 & 1.1 & 1600 & 2200 \\
\hline $7,0.125,16$ & 5 & 5.2 & 6.6 & -19.0 & 0.14 & 0.26 & 0.47 & 260 & 360 \\
\hline $8,0.125,16$ & 5 & 5.3 & 6.7 & -12.3 & 0.061 & 0.11 & 0.23 & 58 & 80 \\
\hline $9,0.125,16$ & 5 & 5.4 & 6.9 & -6.4 & 0.029 & 0.055 & 0.12 & 15 & 22 \\
\hline $10,0.125,16$ & 5 & 5.6 & 7.1 & -1.25 & 0.015 & 0.029 & 0.070 & 4.8 & 6.8 \\
\hline $7,0.25,8$ & 5 & 6.0 & 7.7 & 7.8 & 0.0026 & 0.0054 & 0.0082 & 0.66 & 0.97 \\
\hline $13,0.125,24$ & 7 & 6.4 & 7.4 & -19.2 & 0.28 & 0.50 & 0.66 & 360 & 450 \\
\hline $16,0.125,24$ & 7 & 7.0 & 8.4 & -4.2 & 0.035 & 0.070 & 0.13 & 13 & 18 \\
\hline
\end{tabular}


Table 2 Performance indices for the FE beam patterns of the examples (end-fire beam)

\begin{tabular}{llllllllll}
\hline Parameters $(M, 4, N)$ & Number of beam patterns & $G_{E}(3 \mathrm{D})(\mathrm{dB})$ & $G_{E}(2 \mathrm{D})(\mathrm{dB})$ & $G_{n}(\mathrm{~dB})$ & $\gamma_{E}(3 \mathrm{D})$ & $\gamma_{E}(2 \mathrm{D})$ & $\gamma_{n}$ & $Q_{\mathrm{bw}}(3 \mathrm{D})$ & $Q_{\mathrm{bw}}(2 \mathrm{D})$ \\
\hline $9,0.1,21$ & 5 & 12.2 & 8.4 & -5.3 & 0.069 & 0.036 & 0.11 & 56 & 24 \\
$8,0.25,12$ & 7 & 14.0 & 9.3 & -1.1 & 0.041 & 0.022 & 0.061 & 32 & 11 \\
$3,0.25,4$ & 3 & 7.4 & 5.8 & 3.0 & 0.011 & 0.0066 & 0.014 & 2.8 & 1.9 \\
$5,0.125,8$ & 3 & 7.7 & 6.0 & 4.2 & 0.011 & 0.0065 & 0.019 & 2.25 & 1.51 \\
$5,0.125,16$ & 5 & 12.7 & 8.7 & -24.1 & 0.43 & 0.22 & 0.71 & 4700 & 1900 \\
$6,0.125,16$ & 5 & 12.5 & 8.6 & -14.8 & 0.16 & 0.087 & 0.27 & 540 & 220 \\
$7,0.125,16$ & 5 & 12.4 & 8.5 & -8.2 & 0.082 & 0.044 & 0.13 & 110 & 47 \\
$8,0.125,16$ & 5 & 12.2 & 8.4 & -3.2 & 0.048 & 0.026 & 0.079 & 34 & 14 \\
$9,0.125,16$ & 5 & 11.9 & 8.3 & 0.79 & 0.031 & 0.017 & 0.051 & 13 & 5.6 \\
$10,0.125,16$ & 5 & 11.6 & 8.1 & 4.0 & 0.021 & 0.012 & 0.035 & 5.8 & 2.6 \\
$7,0.25,8$ & 5 & 10.1 & 7.2 & 7.8 & 0.01 & 0.0064 & 0.0082 & 1.7 & 0.87 \\
$13,0.125,24$ & 7 & 8.2 & 8.1 & -11.8 & 0.071 & 0.037 & 0.11 & 67 & 22 \\
$16,0.125,24$ & 7 & 8.1 & 8.1 & 0.4 & 0.027 & 0.015 & 0.041 & 7.6 & 2.7 \\
\hline
\end{tabular}

radiation resistance of the elements tends to be smaller than in a conventional array [12, Chap. 7], and it may therefore be necessary to resort to unconventional technology to mitigate the impact of thermal dissipation. It is also worth pointing out that the approximations inherent in the FE procedure (notably in (11)) limit the extrapolation range and the quality of the corresponding super-directive beam patterns. Most of the above issues are discussed in Section 3, where we finally end up with some design guidelines.

\section{Discussion of performance factors}

The main aspects to be considered for the assessment of the FE multiple beamformer defined by the rows of $\overline{\mathbf{F}}_{i}^{-1}$ or $\overline{\mathbf{F}}_{i}^{\dagger}$ in (16)-(17) are as follows: (1) the shape and regularity of beam patterns, in particular their suitability to cover the entire horizon without significant overlap; (2) the array gain associated to each beam, i.e., the improvement it warrants, with respect to an isotropic antenna, in the ratio between the useful signal power, supposed to arrive from the direction of maximum beam response, and that of the external noise: (3) the self-noise gain associated to each beam, i.e., the ratio between the useful signal power at the beam output and that of internal noise, generated by the dissipative components of the receiver; (4) the sensitivity of the above parameters to errors in the implementation of the array and the beamforming weights; (5) the bandwidth of each beam.

\subsection{FE beam patterns}

We denote as $\mathbf{w}_{k}$ the $k$ th row of the matrix $\overline{\mathbf{F}}_{i}^{-1}$ or $\overline{\mathbf{F}}_{i}^{\dagger}$, taken for convenience with normalized norm: $\left\|\mathbf{w}_{k}\right\|=1$, $k=0,1, \cdots, Q-1$. Each of these rows defines a beam pattern, as follows

$$
g_{k}(\phi)=\mathbf{w}_{k}^{H} \mathbf{u}(\phi), \quad k=0,1, \cdots, Q-1
$$

where $\mathbf{u}(\phi)$ is the unit-norm vector associated to the direction $\phi$ (see Fig. 2)

$\mathbf{u}(\phi) \triangleq \frac{1}{\sqrt{N}}\left(\begin{array}{lllll}1 & e^{-j 2 \pi \Delta \cos \phi} & e^{-j 2 \pi 2 \Delta \cos \phi} & \cdots & e^{-j 2 \pi(N-1) \Delta} \cos \phi\end{array}\right)^{t}$

Recalling how the weighing vectors were obtained, it is expected that the associated beam patterns are similar, albeit not identical, to those that would be obtained from a vector of $N$ actual array elements, and the more so the less the required extrapolation effort, in particular, the smaller the difference $N-M$. This conjecture was confirmed by extensive evaluations, which showed that for moderate extrapolation length the FE beams have a regular shape with a main lobe and moderate secondary lobes. Also, the main lobes are arranged in the angular domain so as to cover the entire horizon uniformly, and their orientation and angular width are such that they intersect approximately at the same level. Examples of beam patterns obtained through the described approach are presented and discussed in Section 4.

\subsection{Array gain vs. external noise}

We recall from our earlier discussions that samples of the noise field taken by the array elements are generally correlated. One exception is the 3D isotropic uncorrelated noise with half-wavelength spaced elements (see (3)). When the noise field is 2D horizontal isotropic then (5) holds, i.e., the noise field is correlated even when the elements are spaced by half-wavelength. In any case, the noise field is correlated if the elements are spaced tightly $(\Delta<1 / 2)$. Curiously this aspect is often overlooked in the literature, notably when formulating the noise vector 
model at the output of a receiving array, ${ }^{8}$ but actually it cannot be neglected since it explains why the gain of a fixed-length uniformly weighted ULA with $\Delta<1 / 2$ does not grow with the number of elements, while conversely it warrants the achievement of large array gains in super-directive regime. Given a $M$-element ULA with weights $\mathbf{w}=\left(\begin{array}{lllll}w_{0} & w_{1} & \cdots & w_{M-1}\end{array}\right)^{t}$, we define the array gain $G_{E}$ with respect to the external noise as the inverse of the ratio between the beam output power due to external noise sources and the noise power that would be obtained at the output of an isotropic antenna having in all directions a response equal to the maximum beam response. In this way $G_{E}$ also represents the increase in signal-to-external-noise ratio when the signal source is on the beam steering direction. As is known, the above definition leads in general to a result depending on the relative angular distribution of noise sources with respect to the beam steering direction. Exceptions to this rule are for the two limiting cases of isotropic 3D and 2D angular noise distributions considered at points (1) and (2) of Section 2, for which the array gain turns out to be respectively [12]:

Array gain vs. $3 D$ isotropic noise

$$
G_{E}=\frac{\mathbf{w}^{H} \mathbf{P}\left(\Delta, \phi_{0}\right) \mathbf{w}}{\mathbf{w}^{H} \mathbf{S}(\Delta) \mathbf{w}}
$$

Array gain vs. $2 D$ isotropic noise ${ }^{9}$

$$
G_{E}=\frac{\mathbf{w}^{H} \mathbf{P}\left(\Delta, \phi_{0}\right) \mathbf{w}}{\mathbf{w}^{H} \mathbf{J}_{0}(\Delta) \mathbf{w}}
$$

where $\phi_{0}$ is the steering direction and $\mathbf{P}, \mathbf{S}$, and $\mathbf{J}_{0}$ are Hermitian $M \times M$ matrices, with entries

$$
\begin{aligned}
& {\left[\mathbf{P}\left(\Delta, \phi_{0}\right)\right]_{i, k}=e^{-j 2 \pi(i-k) \Delta \cos \phi_{0}}} \\
& {[\mathbf{S}(\Delta)]_{i, k}=\operatorname{sinc}[2(i-k) \Delta]} \\
& {\left[\mathbf{J}_{0}(\Delta)\right]_{i, k}=J_{0}[2 \pi(i-k) \Delta]}
\end{aligned}
$$

The gains achievable through the above approach are evaluated numerically for some examples in Section 4.

\subsection{Array gain vs. internal noise}

When the array is used in reception, each element is connected to front-end hardware affected by self noise that can be modeled as AWGN with two-sided power spectral density $2 N_{0}{ }^{10}$ The contribution of these internal sources of noise to the beam output is a combination of IID processes weighted by the beamformer coefficients. As we have done for the external noise, we are interested to formulate a reasonable definition of the array gain with respect to the internal noise. As already pointed out, most literature on MIMO systems does not distinguish between external and internal noise, although their statistical behavior is different and the definition of array gain in a strict sense should only take into account the external sources of disturbance. In any case, the mechanisms through which the two mentioned types of noise-internal and external-impact the system performance are definitely different, and for our purposes, it seems adequate to treat them separately.

As is known, a uniform-weighing beamformer has an intrinsic capability to combat the effects of internal noise since the useful signal components at the output of the array elements add coherently, while the noise terms add up incoherently. Instead a super-directive array tends to reduce the signal level compared to that of self noise in view of the sign variations occurring in the beamformer weights (an example is given in Section 2.4), and this tendency must be carefully kept under control by a proper choice of the design parameters, so as to ensure the array feasibility.

To proceed, we compare the performance of a receiver employing an $M$ - element array antenna with that of a receiver relying on a single-element antenna. Denoting as $\mathrm{SNR}^{(1)}=E_{s} / N_{0}^{(1)}$, the received-energy-per-symbol-toself-noise-spectral-density ratio for the single-element antenna, it is easily found that this ratio at the output of a beam employing the weighing vector $\mathbf{w}$ with steering direction $\phi_{0}$ coincident with the signal DOA becomes

$$
\mathrm{SNR}^{(M)}=\frac{E_{s}}{N_{0}^{(M)}} \frac{\mathbf{w}^{H} \mathbf{P}\left(\Delta, \phi_{0}\right) \mathbf{w}}{\mathbf{w}^{H} \mathbf{w}}
$$

where $\mathbf{P}\left(\Delta, \phi_{0}\right)$ is given by (23) and $N_{0}^{(M)}$ is the equivalent self-noise spectral density of each receiving channel of the array $^{11}$ and represents a design parameter, not necessarily to be taken equal to $N_{0}^{(1)}$. We observe that in (26) $E_{s}$ denotes the received signal energy at the output of the generic array element; and hence, it is the same physical parameter involved in the definition of $\mathrm{SNR}^{(1)}$.

It seems reasonable to require that $\mathrm{SNR}^{(M)} \gtrsim \mathrm{SNR}^{(1)}$, i.e., that the beamformer does not deteriorate the signalto-self-noise ratio with respect to the single-element antenna receiver. This leads to the design criterion of choosing the self-noise level for each array channel such that the following condition holds

$$
N_{0}^{(M)} \lesssim N_{0}^{(1)} G_{n}
$$

where $G_{n}$ is the self-noise gain, defined as

$$
G_{n} \triangleq \frac{\mathbf{w}^{H} \mathbf{P}\left(\Delta, \phi_{0}\right) \mathbf{w}}{\mathbf{w}^{H} \mathbf{w}}
$$

Actually, the index $G_{n}$ (also referred to in the literature with different names, e.g., white noise gain in [13, p. 1366], [17] and [21]) can be regarded as a margin offered by the array to counteract its own 
noise, that can be exploited to reduce complexity and cost of the front-end low-noise amplifiers. Now, while for a uniform-weighing beamformer the parameter $G_{n}$ is indeed greater than unity (and equal to $M$ for the specific case of uniform-weighing vector), thus representing a true gain, instead for a super-directive array $G_{n}$ may occur to be less than one, and even far smaller than one as the array super-directive properties get more and more pronounced. For instance, it is found that the first row of the matrix (18), relevant to the broadside beam, yields $G_{n} \approx-17.5 \mathrm{~dB}$, a very small value, possibly incompatible with other design constraints. In fact, it is apparent that taking $G_{n}$ less than unity entails a more stringent requirement on the self noise of the array elements in comparison with the single-element antenna receiver, and this seems acceptable only if the implied technological effort is reasonably mild, say of the order of a few decibels. This is one of the criteria to be taken up again in Section 4.

\subsection{Sensitivity to array imperfections}

To assess the sensitivity of the main performance indices to small perturbations $\delta \mathbf{w}$ occurring in the implementation of the beamformer-weighing vector (perturbations to which, up to a first approximation, we will also ascribe the impact of inaccuracies in the mechanical positioning of the array elements), we expand the above indices into a Taylor series around the nominal weights, truncated at the linear terms. We then proceed carrying out a statistical error analysis, under the assumption that the perturbations can be modeled as random variables. Starting from expression (21) of the 3D array gain, we have to evaluate ([13, Sect. A.7.4], [22, Eq. (2.8)])

$$
\begin{aligned}
\delta G_{E} \approx & {\left[\begin{array}{ll}
\frac{\partial G_{E}}{\partial \mathbf{w}^{t}} & \frac{\partial G_{E}}{\partial \mathbf{w}^{* t}}
\end{array}\right]\left[\begin{array}{c}
\delta \mathbf{w} \\
\delta \mathbf{w}^{*}
\end{array}\right]=G_{E}\left(\frac{\mathbf{w}^{H} \mathbf{P}}{\mathbf{w}^{H} \mathbf{P} \mathbf{w}}-\frac{\mathbf{w}^{H} \mathbf{S}}{\mathbf{w}^{H} \mathbf{S} \mathbf{w}}\right) \delta \mathbf{w} } \\
& +G_{E}\left[\frac{\left(\mathbf{w}^{H} \mathbf{P}\right)^{*}}{\mathbf{w}^{H} \mathbf{P} \mathbf{w}}-\frac{\left(\mathbf{w}^{H} \mathbf{S}\right)^{*}}{\mathbf{w}^{H} \mathbf{S} \mathbf{w}}\right] \delta \mathbf{w}^{*}
\end{aligned}
$$

where the derivative with respect to $\mathbf{w}$ is taken under the assumption that $\mathbf{w}^{*}$ remains constant and vice versa, and we dropped the dependency on $\Delta$ and $\phi_{0}$. It follows that

$$
\frac{\delta G_{E}}{G_{E}} \approx 2 \operatorname{Re}\left\{\mathbf{b}^{H} \delta \mathbf{w}\right\}
$$

where

$$
\begin{aligned}
& \mathbf{b}^{H} \triangleq \mathbf{w}^{H} \mathbf{B} \\
& \mathbf{B} \triangleq \frac{\mathbf{P}}{\mathbf{w}^{H} \mathbf{P} \mathbf{w}}-\frac{\mathbf{S}}{\mathbf{w}^{H} \mathbf{S} \mathbf{w}}
\end{aligned}
$$

As expected, for small perturbations of the beamforming weights, the relative variation of $G_{E}$ turns out to be a combination of the relative variations of the numerator and the denominator of (21). Assuming for the components of $\delta \mathbf{w}$ a complex-valued IID Gaussian model with zero mean and variances of the real and imaginary components all equal to $\sigma_{w}^{2}$, after some elaboration, we are led to

$$
\frac{E\left\{\delta G_{E}\right\}}{G_{E}} \approx 0 ; \frac{E\left\{\delta G_{E}^{2}\right\}}{G_{E}^{2}} \approx 4 \sigma_{w}^{2}\|\mathbf{b}\|^{2}
$$

where from (31)

$$
\|\mathbf{b}\|^{2}=\mathbf{b}^{H} \mathbf{b} \triangleq \mathbf{w}^{H} \mathbf{B B}^{H} \mathbf{w}
$$

Denoting as $\quad \overline{\mathbf{w}}=\mathbf{w} /\|\mathbf{w}\|$ the unit-norm-weighing vector, the relative RMS deviation of $G_{E}$ in (33) can be written as

$$
\gamma_{E}=\frac{\sqrt{E\left\{\delta G_{E}^{2}\right\}}}{G_{E}} \approx 2 \sigma_{\bar{w}}\|\overline{\mathbf{b}}\|
$$

with $\sigma_{\bar{w}}^{2}=\sigma_{w}^{2} /\|\mathbf{w}\|^{2}$ and

$$
\|\overline{\mathbf{b}}\|^{2}=\|\mathbf{b}\|^{2}\|\mathbf{w}\|^{2}=\overline{\mathbf{w}}^{H} \overline{\mathbf{B}} \overline{\mathbf{B}}^{H} \overline{\mathbf{w}}
$$

and

$$
\overline{\mathbf{B}}=\mathbf{B}\|\mathbf{w}\|^{2}=\frac{\mathbf{P}}{\overline{\mathbf{w}}^{H} \mathbf{P} \overline{\mathbf{w}}}-\frac{\mathbf{S}}{\overline{\mathbf{w}}^{H} \mathbf{S} \overline{\mathbf{w}}}=\frac{\mathbf{P}}{G_{n}}-\frac{G_{E}}{G_{n}} \mathbf{S} .
$$

Following the same line of reasoning, the relative RMS deviation of the gain $G_{E}$ over the 2D external isotropic noise is again given by (35)-(37), the only difference being that the matrix $\mathbf{S}$ in (37) is replaced by $\mathbf{J}_{0}(\Delta)$ given by (25). Finally, a similar result applies to the self-noise gain (28), for which the matrix $\mathbf{S}$ in (37) is replaced by the identity matrix, leading to

$$
\gamma_{n}=\frac{\sqrt{E\left\{\delta G_{n}^{2}\right\}}}{G_{n}} \approx 2 \sigma_{\bar{w}}\left\|\overline{\mathbf{b}}_{n}\right\|
$$

where

$$
\left\|\overline{\mathbf{b}}_{n}\right\|^{2}=\overline{\mathbf{w}}^{H} \overline{\mathbf{B}}_{n} \overline{\mathbf{B}}_{n}^{H} \overline{\mathbf{w}}=\frac{\overline{\mathbf{w}}^{H} \mathbf{P} \mathbf{P}^{H} \overline{\mathbf{w}}}{\left(\overline{\mathbf{w}}^{H} \mathbf{P} \overline{\mathbf{w}}\right)^{2}}-1
$$

Noting that $\mathbf{P} \mathbf{P}^{H}=M \mathbf{P}$, from (38)-(39) we find

$$
\gamma_{n} \approx 2 \sigma_{\bar{w}}\left\|\overline{\mathbf{b}}_{n}\right\|=2 \sigma_{\bar{w}} \sqrt{\frac{M}{G_{n}}-1}
$$

This result indicates that the relative RMS variation of the self-noise gain in response to a relative RMS variation $\sqrt{2} \sigma_{\bar{w}}$ of the normalized weights is proportional to the latter variation, the proportionality factor being greater than unity when $G_{n}<2 M / 3$. 
Collecting the above, the sensitivity of the array gains to perturbations of the weighing vector is given by (35) and (40). These equations represent useful design tools when dealing with super-directive arrays since the relative errors occurring in the implementation of the beamformer weights usually map onto amplified relative errors on the array gains. Therefore, use of these equations allows us to identify the degree of super-directivity that can be safely assigned to the array without having to tackle substantial feasibility issues. For instance, using as beamforming vector, the first row of matrix (18), we find that the broadside array gains with respect to the $2 \mathrm{D}$ and $3 \mathrm{D}$ isotropic noises are 6.8 and $5.4 \mathrm{~dB}$, respectively, while the self-noise gain is $-17.5 \mathrm{~dB}$. This pronounced super-directivity reflects in a rather high sensitivity to array imperfections: actually, using (35) and (40), we find from simulations that a $1 \%$ relative RMS perturbation on the beamformer weights entails relative RMS variations close to 33 and $27 \%$ for the 2D and $3 \mathrm{D}$ array gains, respectively, and even larger deviations for $G_{n}$. To limit the impact on the array gains to just a few percent, we should constrain array imperfections not to exceed say $0.1 \%$ or so, a rather demanding requirement. The design criteria for jointly obtaining adequate super-directive properties and robustness against array imperfections are discussed in Section 4.

\subsection{Bandwidth}

A further aspect to be considered when dealing with FE super-directive arrays is the sensitivity of the array response to a mismatch of the frequency of signals actually handled by the arrays with respect to the nominal array (center) frequency. Indeed, it is well known that super-directivity can greatly restrict the array fractional bandwidth, defined as the ratio between the actual bandwidth and the array nominal center frequency. It is therefore appropriate, to avoid signal distortion, that the bandwidth of the signals received or transmitted by the array is smaller than the array bandwidth. The array fractional bandwidth for 3D isotropic reception is the inverse of the $\mathrm{Q}_{\mathrm{bw}}$ factor given in [12] Eq. (9.23)] as

$$
Q_{\mathrm{bw}} \triangleq \frac{\mathbf{w}^{H} \mathbf{w}}{\mathbf{w}^{H} \mathbf{S}(\Delta) \mathbf{w}}
$$

which, recalling (21) and (28), also reads as

$$
Q_{\mathrm{bw}}=\frac{G_{E, 3 \mathrm{D}}}{G_{n}}
$$

where we designate as $G_{E, 3 \mathrm{D}}$ the array gain vs. the $3 \mathrm{D}$ isotropic noise. A result similar to (42) also applies when the parameter $Q_{b w}$ is defined with reference to $2 D$ isotropic reception, with $G_{E, 3 \mathrm{D}}$ replaced by the $2 \mathrm{D}$ array gain. With reference to the example in Section 2.3, from (42) we find for the broadside beam $Q_{b w} \approx 200$, i.e., a fractional bandwidth around $0.5 \%$. For the 2D case, we get $Q_{\mathrm{bw}} \approx 270$, or a fractional bandwidth close to $0.4 \%$. If the center frequency is in the order of a few gigahertz, this implies a bandwidth around a few tens megahertz. Larger bandwidths can be attained with different choices of the design parameters (see Tables 1 and 2 in Section 4), such to ensure compatibility with existing wireless standards. In other application areas, such as aeroand underwater acoustics, the relative bandwidths of signals may occur to be so large as to enforce the use of complex equalization techniques in conjunction with super-directive arrays $[17,23]$. In this respect, it is noted that end-fire beams seem to offer improved robustness and larger bandwidths than broadside beams (see discussion in [23] and results in Section 4.2).

\section{Beamformer design}

\subsection{Design criteria}

Based on the results in the foregoing sections, we now outline a procedure for the design of a FE-based superdirective multiple beamformer and for assessment of its practical feasibility. We start by fixing the normalized effective array length $L$, a parameter commonly subject to practical limitations. Given the number of array elements $M$ and the inter-element spacing $\Delta$, such that $L=M \Delta$, the basis directions associated to the array are

$$
\bar{\Omega}_{k} \triangleq \cos \bar{\phi}_{k}=\frac{k}{L}=\frac{1}{\Delta} \frac{k}{M}, \quad k=-\left\lceil\frac{M}{2}-1\right\rceil, \cdots-1,0,1, \cdots,\left\lfloor\frac{M}{2}\right\rfloor
$$

among which the real directions are those satisfying $|k| /$ $(\Delta M) \leq 1$, whose number is $1+2\lfloor\Delta M\rfloor$. To achieve extrapolation and related angular super-resolution, we have to select a number $N>M$ of elements of a longer virtual ULA incorporating the actual array as described in Section 2.2. The basis directions associated to this array are given by (7), and the number of real directions is $Q=1+2\lfloor\Delta N\rfloor$, that we require to exceed that relevant to the actual array, i.e., $\lfloor\Delta N\rfloor>\lfloor\Delta M\rfloor$, so as to achieve super-resolution. A further condition is given by (12), allowing to solve the system of equations (13). Summing up, we have the following constraints

$$
\begin{aligned}
& \lfloor\Delta N\rfloor>\lfloor\Delta M\rfloor \\
& 1+2\lfloor\Delta N\rfloor \leq M
\end{aligned}
$$

From (45), we also find $\left\lfloor\Delta N_{\rfloor} \leq(M-1) / 2\right.$ and substituting in (44) produces

$$
\frac{M-1}{2} \geq\lfloor\Delta N\rfloor>\lfloor\Delta M\rfloor
$$

confirming that to achieve angular super-resolution, it is strictly necessary that $\Delta<1 / 2$. Use of (46) permits to 
identify the lower and upper limits for the length $N$ of the extrapolated vector. For the example in Section 2.2, it is easily found that these limits are $N_{\min }=10$ and $N_{\max }=49$, respectively. However, not all values of $N$ in this wide interval are acceptable in view of their impact on the array self-noise gain and array response sensitivity to perturbations in the weighing vector, as discussed in Section 3. As we already observed, the choice $N=21$ leads to severe implementation issues, that tend to worsen further at larger values of $N$.

Collecting the above, a possible approach to search good trade-off solutions in the design of a FE-based super-directive multiple beamformer is as follows:

1. Given the effective length $L$, choose the number $M$ of elements such that $\Delta<1 / 2$, keeping in mind that the larger $M$ is, i.e., the more demanding is the array implementation, the easier is to achieve super-directive properties from the array, as well as robustness to self noise and implementation errors;

2. From (46), find the range of possible design values for $N$ and select $N$ as close as possible to $N_{\min }$ and such to achieve the desired number $Q=1+2\lfloor\Delta N\rfloor$ of super-directive beams, taking into account that the beamformer implementation complexity grows with $Q$; a further condition that may be desirable to introduce is that $N$ is an integer multiple of $1 / \Delta$, so as to achieve two beams pointed exactly at the end-fire directions (see (7));

3. Calculate the unit-norm weighing vectors of the FE multiple beamformer from the rows of the matrices $\overline{\mathrm{F}}_{i}^{-1}$ or $\overline{\mathrm{F}}_{i}^{\dagger}$;

4. For each weighing vector, from (21) or (22), evaluate the array gain $G_{E}$ and the associate beam pattern;

5. For each weighing vector, from (28) and (35), (38), (42) verify that the self-noise gain, the beam sensitivity to array perturbations and the bandwidth take on affordable values.

It was found that the procedure outlined above can lead to a significant increase of the number of approximately orthogonal beams, in comparison with a uniformly weighted array of equal length. On the other hand, beyond a certain degree of super-directivity, the FE beam patterns may suffer from excessive degradation in view of the approximations inherent in (11). The above properties are discussed further in the next section by means of specific examples.

It is also worth observing that, for a given number and spacing of the array elements, the beams synthesized by means of the above approach do not generally achieve the largest possible amount of (super-) directivity, i.e., the maximum array gain vs. external noise for a fixed array response in the steering direction. The upper limit to this gain is established by the Capon weighing window [13, Sect. 6.2.1.1], but application of this window without additional constraints often leads to unsatisfactory results, e.g., to beam patterns with very large side lobes. However, as shown in the examples of Section 4.2, the above limit can be closely approached using an adequate extrapolation effort.

\subsection{Examples}

We limit our consideration to arrays of small length, up to about $2 \lambda$. To fix a benchmark, Fig. $4 a-d$ shows the sets of beam patterns associated to the (all real) basis directions of standard uniform-weighing $\lambda / 2$-spaced arrays of physical lengths $L_{\mathrm{a}}=\lambda / 2, \lambda, 3 \lambda / 2,2 \lambda$ (i.e., with $M=2,3$, $4,5)$, respectively. The patterns are plotted using different line styles. As is known, for even $M$, one of the patterns exhibits two antipodal main lobes looking at the end-fire directions, while for odd $M$, the end-fire directions are not included in the set (43). Table 3 shows the number of beams and the array gains at broadside achievable for these arrays. Also shown are the array gains for the endfire beams where applicable.

Turning to FE beamforming, we consider first the case $M=3, \Delta=1 / 4$ yielding an actual array length $L_{\mathrm{a}}=\lambda / 2$. In this case, from (43), it is seen that a single real basis direction exists (broadside). Application of the procedure outlined above leads to a single compatible value of $N$, namely $N=4$, yielding the set of three beam patterns depicted in Fig. 5a, that should be compared with the two beams in Fig. 4a. These three beams are slightly super-directive as is confirmed by the self-noise gain of $3 \mathrm{~dB}$ (see Tables 1 and 2), but they do not pose feasibility issues.

In an attempt to further boost the array directivity, we may select smaller values for $\Delta$ without changing the array length. Letting $M=5, \Delta=1 / 8$, we find $N_{\min }=8, N_{\max }=16$; using $N=8$ yields the three beams of Fig. $5 \mathrm{~b}$, which are quite similar to those in Fig. 5a, except some reduction in the side lobe level, and related beneficial impact on the performance indices (Tables 1 and 2). Here, the gain of broadside and end-fire beams vs. the 3D isotropic noise is 3.0 and $7.7 \mathrm{~dB}$, respectively. For comparison, from [13, Eqs. (6.14)-(6.15)], we find that the gain achieved by the Capon beamformer for the same values of $M$ and $\Delta$ in the same directions is 5.5 and $13.8 \mathrm{~dB}$, respectively, i.e., 2.5 and $6.1 \mathrm{~dB}$ higher than those achieved by the FE beamformer, thus indicating the existence of sizable margins to further increase the array directivity. Actually, if we let $N=16$ instead of $N=8$, we are led to the five beams depicted in Fig. 5c achieving a substantial directivity boost. However, even ignoring the presence of significant side lobes for the broadside beam pattern, these sharper beams prospect severe feasibility issues, as the system sensitivity to self noise and other 


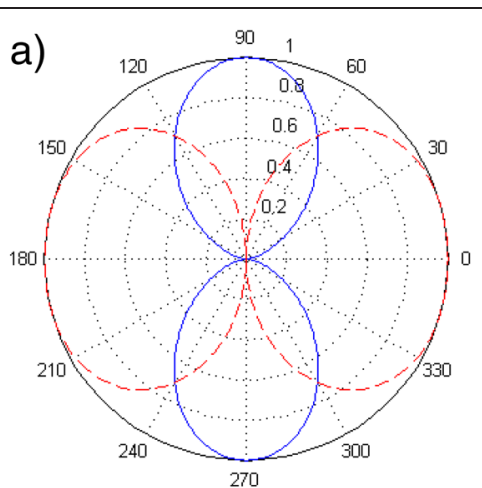

c)

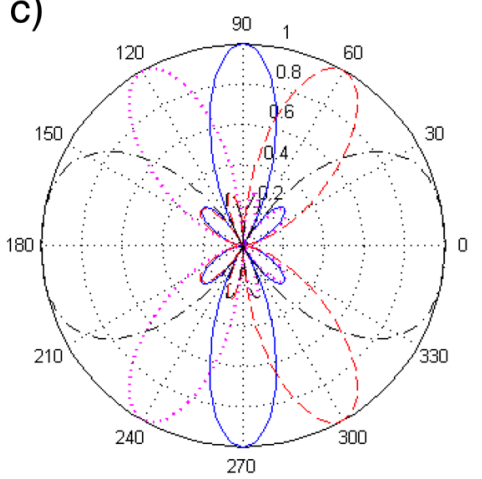

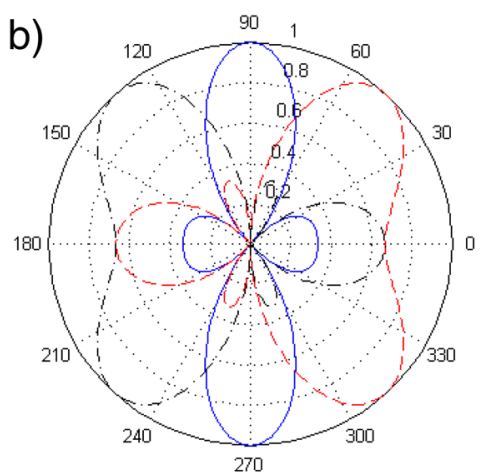

d)

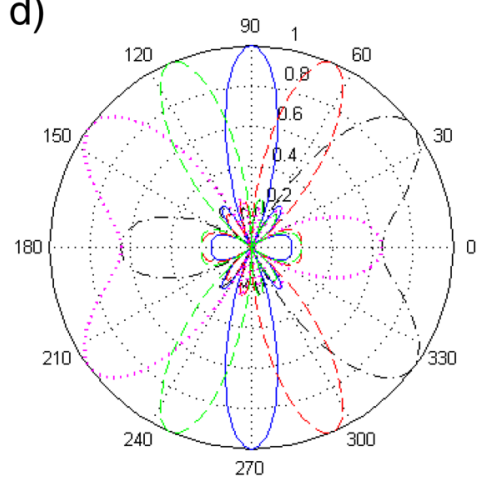

Fig. 4 Uniform-weighted array $(\Delta=0.5)$. Beam patterns for a $M=2$, b $M=3, \mathbf{c} M=4$, d $M=5$

perturbations grows considerably (see Tables 1 and 2, showing that the self-noise gain at broadside and end-fire drops down to -37.5 and $-24.1 \mathrm{~dB}$, respectively!). It is worth pointing out that in this case, the gains of broadside and end-fire beams vs. the $3 \mathrm{D}$ isotropic noise turn out to be 5.0 and $12.7 \mathrm{~dB}$, respectively, i.e., fairly close to the performance of the Capon beamformer (the distance is now 0.5 and $1.1 \mathrm{~dB}$ for broadside and end-fire, respectively). Similar results and conclusions apply to the $2 \mathrm{D}$ noise gains.

To mitigate the effect of self noise, we can slightly increase the array length, adding elements without changing their spacing. Retaining $\Delta=1 / 8, N=16$ and selecting $M=6,7,8$ leads to the patterns of Fig. $6 \mathrm{a}-\mathrm{c}$ that look all similar to Fig. 5c, but actually their feasibility constraints get looser and looser with $M$. For instance, the self-noise gain for the broadside beam pattern of Fig. $6 \mathrm{c}$ is $-12.3 \mathrm{~dB}$ (Table 1), and it could be further increased to around -6.4 and $-1.3 \mathrm{~dB}$ by letting $M=9$ and $M=10$, respectively. In these conditions, the FE approach permits to approximately double the array length (from around $M=8 \div 10$ to $N=16$, i.e., to obtain a virtual array length close to $2 \lambda$ from an actual length around $\lambda$.

This property was found to hold true for longer arrays as well. In general, the FE technique seems to permit, with reasonable effort and good quality of the resulting beam patterns, the achievement of virtual lengths exceeding the actual size by approximately one wavelength or slightly more. This is not surprising, however, as similar results are mentioned in the literature dealing with band-limited signal extrapolation. Indeed, in [9], it is shown that a band-limited signal after truncation in the time domain can be extrapolated, with reasonable effort and accuracy, over an interval exceeding the truncation window by a very few times the inverse of the signal bandwidth. Attempts to do better soon conflict with feasibility issues. From the above, we can argue that the

Table 3 Main performance parameters for standard uniform-weighing $\lambda / 2$-spaced arrays

\begin{tabular}{|c|c|c|c|c|c|c|}
\hline Parameters $(M, \Delta)$ & Number of beam patterns & $G_{E}(3 D)(d B)$ broadside & $G_{E}(2 \mathrm{D})(\mathrm{dB})$ broadside & $G_{E}(3 \mathrm{D})(\mathrm{dB})$ end-fire & $G_{E}(2 \mathrm{D})(\mathrm{dB})$ end-fire & $G_{n}(\mathrm{~dB})$ \\
\hline $2,0.5$ & 2 & 3.0 & 4.6 & 3.0 & 1.9 & 3.0 \\
\hline $3,0.5$ & 3 & 4.8 & 6.1 & & & 4.8 \\
\hline $4,0.5$ & 4 & 6.0 & 7.7 & 6.0 & 3.5 & 6.0 \\
\hline $5,0.5$ & 5 & 7.0 & 8.6 & & & 7.0 \\
\hline
\end{tabular}



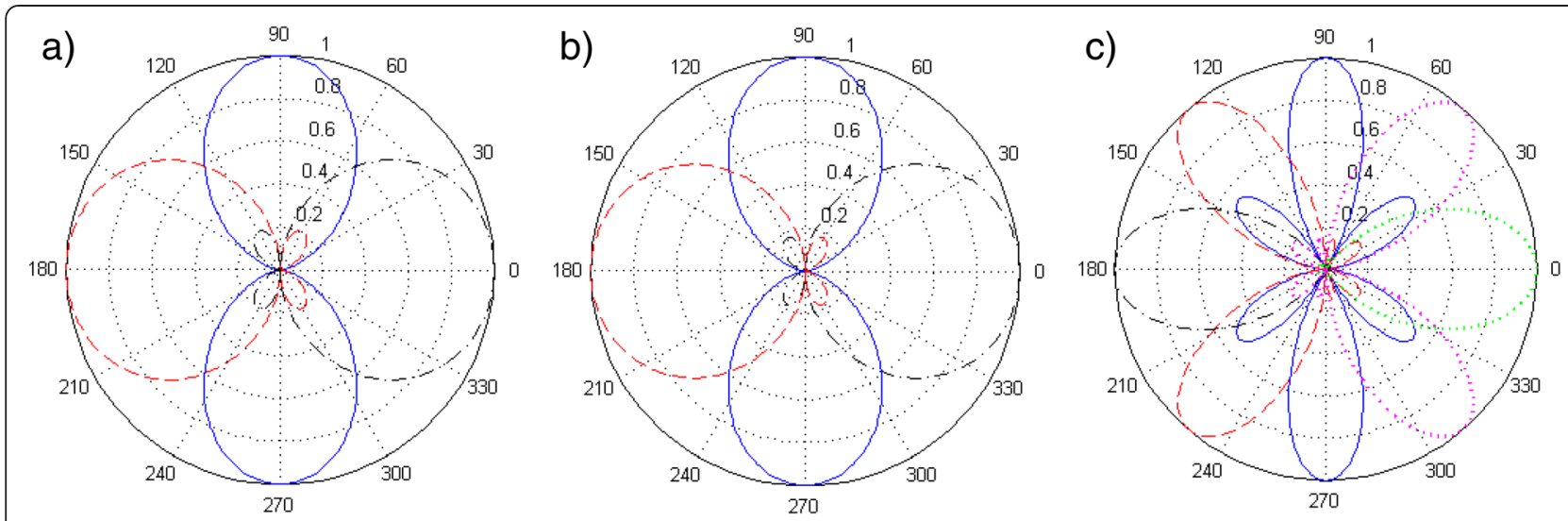

Fig. 5 FE beam patterns for a $M=3, \Delta=0.25, N=4$; b $M=5, \Delta=0.125, N=8$; $\mathbf{c} M=5, \Delta=0.125, N=16$

benefits of FE are especially valuable for relatively small arrays, with size say up to a few wavelengths, when adding a further (albeit virtual) wavelength to the array size produces a significant directivity upgrade.

It is also worth observing (Figs. 4, 5, and 6) that the beam patterns generated by the FE approach are subject to some distortion with respect to the patterns achievable from a standard array of actual length $N$. Notably, some of the FE patterns have more pronounced side lobes and their angular widths and looking directions are somewhat perturbed, even though their arrangement over the horizon appears rather uniform, with adjacent patterns overlapping approximately at the same level. The cited distortions are to be ascribed to the approximations implicit in the FE procedure. We believe they could be mitigated by introducing adjustments in the generation of the weighing windows, but this aspect is not pursued here being out of the paper scope. We limit ourselves to point out that, as far as MIMO applications are concerned, the exact shape of the beam patterns is not of primary interest, a more useful objective being a set of beams uniformly arranged in the angular domain with small overlap on one another. Simulations showed that the correlation coefficient between the outputs of properly designed FE beams in response to a $2 \mathrm{D}$ isotropic noise field does not exceed $0.2 \div 0.3$, not far from the values encountered in conventional multiple beams.

As a further example, we consider an array of physical length $L_{\mathrm{a}}=3 \lambda / 2$, corresponding to $M=4$ for the standard spacing $\Delta=1 / 2$ (Fig. 4c). Using $M=7, \Delta=1 / 4, N=8$ leads to a slightly super-directive set of beams as shown in Fig. 7a. Letting $M=13, \Delta=1 / 8, N=24$ (same actual length as previous) produces the more critical set of beams of Fig. 7b (the self-noise gain at broadside is now $-19.2 \mathrm{~dB}$ ) while, allowing the array to be slightly extended to $M=16, \Delta=1 / 8, N=24$ yields the beams of Fig. $7 \mathrm{c}$, with self-noise gain at broadside around $-4.2 \mathrm{~dB}$. These results substantially confirm that the array can be virtually extended by around one wavelength with reasonable effort. We observe that the solution in Fig. 7c provides seven beams of good quality against the four or five
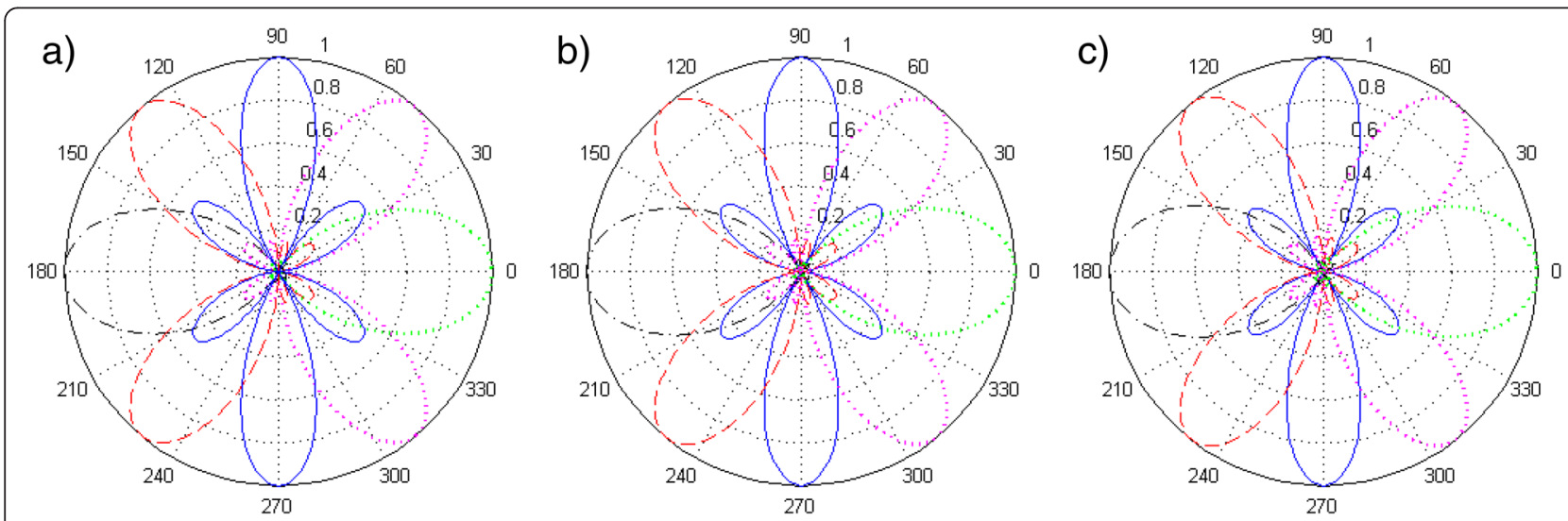

Fig. 6 FE beam patterns for $\Delta=0.125, N=16$. a $M=6, \mathbf{b} M=7, \mathbf{c} M=8$ 

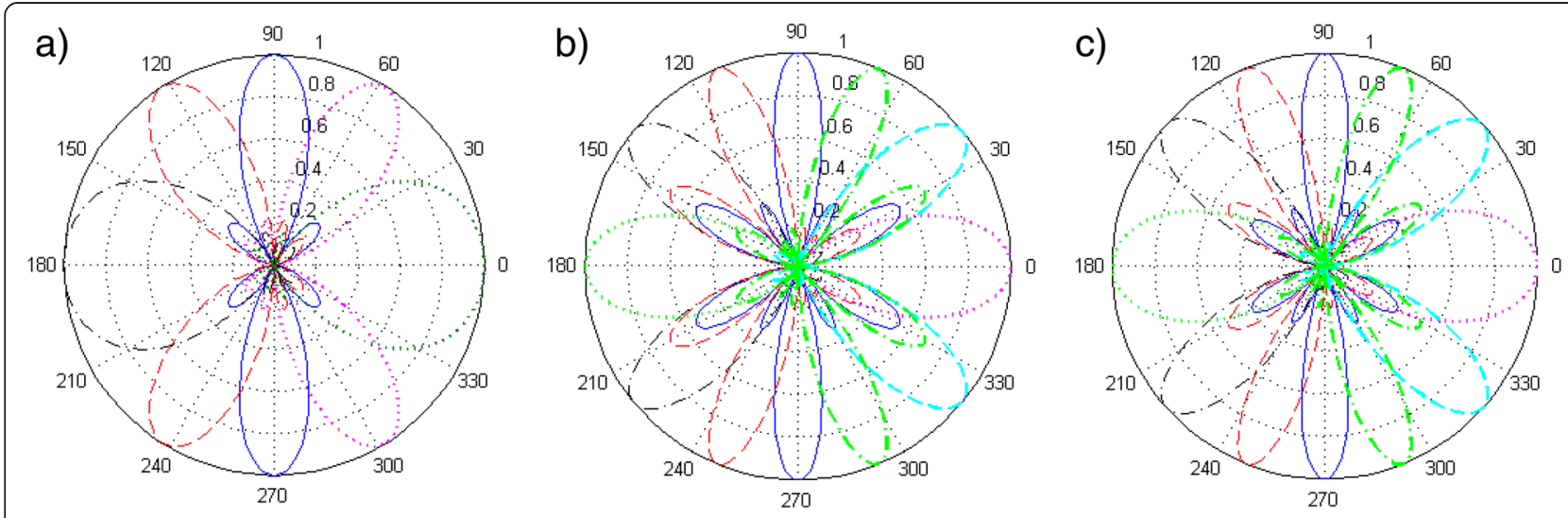

Fig. $7 \mathrm{FE}$ beam patterns for a $M=7, \Delta=0.25, N=8$; b $M=13, \Delta=0.125, N=24$; $\mathbf{c} M=16, \Delta=0.125, N=24$

beams of the uniform-weighing approach shown in Fig. 4 b, c.

Finally, to ease the comparison of results, we summarize in Table 1 the main performance indices for all combinations of FE parameters considered so far, with reference to the beams looking at broadside. Gain margins for off-broadside looking directions are found to be even wider. This is confirmed by Table 2 , showing the values of the same indices for the endfire beams and for the same parameter combinations considered in Table 1.

\section{Conclusions}

It was shown that the bandwidth finiteness of the field sampled by a densely spaced ULA can be exploited to extrapolate the field beyond the array extension and enhance the array angular resolution. One of the results of the paper is that it is possible, with reasonable effort, to achieve accurate extrapolation of the field over a length of the order of a wavelength, equally partitioned at the array ends, thus allowing a significant increase of the virtual array size and its angular discrimination capability when the actual array length is in the order of a few wavelengths or smaller. The proposed technique permits to build approximately orthogonal beams, narrower than those achievable through a conventional uniform-weighing approach, and featuring about the same properties as those obtained from an actual array of length equal to the extrapolation window. In scattering environments, this may boost the spatial multiplexing capability of array antennas for MIMO applications. The price to be paid is an increased implementation complexity of the antenna front-ends, notably the need to arrange more elements within the array spatial aperture, along with a greater sensitivity to self noise and to mechanical and electrical perturbations of the array elements. In the paper, we have indicated design criteria leading to a significant enhancement of array angular resolution with reasonable implementation constraints.

\section{Endnotes}

${ }^{1}$ We define two beams as spatially orthogonal when the cross-correlation of their outputs in response to an isotropic uncorrelated noise field is zero.

${ }^{2}$ The term "uniform" means that the array elements are evenly spaced.

${ }^{3} \mathrm{We}$ observe that, since the array elements are assumed isotropic, the (random uniform) polarization of the received field components is immaterial here.

${ }^{4}$ In more precise terms, the field components generated by all $\infty^{2}$ elements of solid angle making up the 3D horizon are assumed to be zero-mean, of equal intensity, and uncorrelated.

${ }^{5}$ In more precise terms, the field components generated by all $\infty$ angular elements making up the $2 \mathrm{D}$ horizon are assumed to be zero-mean, of equal intensity, and uncorrelated.

${ }^{6}$ Actually the generic element of $\mathbf{u}_{k}$ can be written in the familiar form $\left[\mathbf{u}_{k}\right]_{n}=\exp (-j 2 \pi n k / N) / \sqrt{N}$.

${ }^{7} \mathrm{We}$ momentarily admit that there is no internal noise in the receiving array, deferring a discussion of the impact of internal noise to a later step.

${ }^{8} \mathrm{~A}$ very common assumption in papers dealing with MIMO applications is that the noise vector at the receiving array is uncorrelated, irrespective of the element spacing and of the noise field spatial properties.

${ }^{9}$ The beam maximum response axis is assumed to belong to the horizontal plane for the 2D noise distribution.

${ }^{10} \mathrm{We}$ assume this incorporates all sources of internal noise, from RF to baseband stages as well as the ADC quantization noise.

${ }^{11}$ For simplicity $N_{0}^{(M)}$ is assumed here equal for all channels, but actually the self-noise levels across the 
array could be optimized according to the values assumed for the entries in $\mathbf{w}$.

\section{Abbreviations}

2D, 3D: two- or three-dimensional; ADC: analog-to-digital conversion; AWGN: additive white Gaussian noise; DOA: direction of arrival; FE: field-extrapolation; IID: independent identically distributed; MIMO: multiple-input multiple-output; RF: radio frequency; RMS: root mean square; ULA: uniform linear array.

\section{Competing interests}

The author declares that he has no competing interests.

Received: 28 November 2014 Accepted: 23 July 2015

Published online: 08 August 2015

\section{References}

1. GJ Foschini, Layered space-time architecture for wireless communication in a fading environment when using multiple antennas. Bell Labs Tech. J. 1(2), 41-59 (1996). doi:10.1002/bltj.2015

2. GG Raleigh, JM Cioffi, Spatio-temporal coding for wireless communication. IEEE Trans. Commun. 46(3), 357-366 (1998). doi:10.1109/26.662641

3. D Tse, P Viswanath, Fundamentals of Wireless Communication (Cambridge Univ. Press, New York, 2005)

4. A Papoulis, A new algorithm in spectral analysis and band-limited extrapolation. IEEE Trans. Circ. Sys. 22(9), 735-742 (1975). doi:10.1109/TCS.1975.1084118

5. RW Gerchberg, Super-resolution through error energy reduction. Optica Acta 21(9), 709-720 (1974). doi:10.1080/713818946

6. MS Sabri, W Steenaart, An approach to band-limited signal extrapolation: the extrapolation matrix. IEEE Trans. Circ. Sys. 25(2), 74-78 (1978). doi:10.1109/TCS.1978.1084442

7. JA Cadzow, An extrapolation procedure for band-limited signals. IEEE Trans. Acoust. Sp. Sig. Proc. 27(1), 4-12 (1979). doi:10.1109/TASSP.1979.1163187

8. AK Jain, S Ranganath, Extrapolation algorithms for discrete signals with application in spectral estimation. IEEE Trans. Acoust. Sp. Sig. Proc. 29(4), 830-845 (1981). doi:10.1109/TASSP.1981.1163639

9. HJ Landau, Extrapolating a band-limited function from its samples taken in a finite interval. IEEE Trans. Inform. Theory 32(4), 464-470 (1986). doi:10.1109/TIT.1986.1057205

10. RC Johnson, HA Ecker, JS Hollis, Determination of far-field antenna patterns from near-field measurements. Proc. IEEE 61(12), 1668-1694 (1973). doi:10.1109/PROC.1973.9358

11. AJ Berkhout, D de Vries, J Baan, BW van den Oetelaar, A wave field extrapolation approach to acoustical modeling in enclosed spaces. J. Acoust. Soc. Am. 105(3), 1725-1733 (1999). doi:10.1121/1.426710

12. RC Hansen, Phased Array Antennas (Wiley, New York, 2001)

13. HL Van Trees, Optimum Array Processing-Part IV of Detection, Estimation and Modulation Theory (Wiley, New York, 2002)

14. M Pesavento, YI Abramovich, F Gini, N Sidiropoulos, AM Zoubir, Special issue on advances in sensor array processing in memory of Alex B. Gershman. Signal Process. 93(12), 3361-3588 (2013)

15. DI Lekhovytskiy, YS Shifrin, Statistical analysis of "superresolving" methods for direction-of-arrival estimation of noise radiation sources under finite size of training sample. Signal Process. 93(12), 3382-3399 (2013). doi:10.1016/j.sigpro.2013.03.008

16. SA Vorobyov, Principles of minimum variance robust adaptive beamforming design. Signal Process. 93(12), 3364-3377 (2013). doi:10.1016/ j.sigpro.2012.10.021

17. M Crocco, A Trucco, Design of robust superdirective arrays with a tunable tradeoff between directivity and frequency-invariance. IEEE Trans. Sig. Proc. 59(5), 2169-2181 (2011). doi:10.1109/TSP.2011.2106780

18. ML Morris, MA Jensen, JW Wallace, Superdirectivity in MIMO systems. IEEE Trans. Ant. Propag. 53(9), 2850-2857 (2005). doi:10.1109/TAP.2005.854530

19. RH Clarke, A statistical theory of mobile-radio reception. Bell Sys. Tech. J. 47(6), 957-1000 (1968). doi:10.1002/j.1538-7305.1968.tb00069.x

20. F Krahmer, GE Pfander, P Rashkov, Support Size Conditions for Time-Frequency Representations on Finite Abelian Groups. Tech. Rep. 13, School of Eng. and Science, Jacobs Univ, 2007. http://math.jacobs-university.de/pfander/pubs/ krapfara_tr13.pdf. Accessed 11 Nov 2014
21. H Cox, RM Zeskind, MM Owen, Robust adaptive beamforming. IEEE Trans. Acoust. Sp. Sig. Proc. 35(10), 1365-1376 (1987). doi:10.1109/ TASSP.1987.1165054

22. L Sorber, M Van Barel, L De Lathauwer, Unconstrained optimization of real functions in complex variables. SIAM J. Optim. 22(3), 879-898 (2012). doi:10.1137/110832124

23. A Trucco, F Traverso, M Crocco, Broadband performance of superdirective delay-and-sum beamformers steered to end-fire. J. Acoust. Soc. Am. 135(6), EL331-EL337 (2014). doi:10.1121/1.4879674

\section{Submit your manuscript to a SpringerOpen ${ }^{\circ}$ journal and benefit from:}

- Convenient online submission

- Rigorous peer review

- Immediate publication on acceptance

- Open access: articles freely available online

- High visibility within the field

- Retaining the copyright to your article

Submit your next manuscript at $>$ springeropen.com 